\title{
ABOUT THE COHOMOLOGY RING OF A FINITE ABELIAN GROUP
}

\author{
BY KARL HEINRICH HOFMANN ${ }^{1}$ AND PAUL S. MOSTERT ${ }^{2}$
}

Communicated by Saunders Mac Lane, November 5, 1968

Since the structure of finite abelian groups is so simple, one would expect that the cohomology ring $H(G, R)$ of a finite abelian group $G$ over a commutative ring $R$ to be completely and explicitly known, particularly when the action of $G$ on $R$ is trivial and the ring $R$ is some reasonable ring, say the ring $\boldsymbol{Z}$ of integers. Indeed considerable work has been accomplished in the computation of a homology of finite abelian groups in the context of Eilenberg-MacLane spaces by Eilenberg and MacLane [4]; a remarkable algebraic theory around this topic was built up by Cartan [2]. Further analysis of the homology ring of a finite abelian group is presented in [9]. However, as far as we can see, there still is no explicit and functorial description of $H(G, R)$ in the general case. The following observations still do not fill this gap, but they contribute some new facets and perhaps offer a more direct approach to some results which are in the literature. Our approach is designed specifically to allow for easy generalization to the cohomology ring of a compact abelian group, which we discuss in this journal [6]. The full details and the proofs will appear elsewhere.

Rather than to give too many technical details, we describe the essential features of our approach and try to point out where it differs from other methods.

Each finite abelian group $G$ decomposes into a direct sum of cyclic subgroups $G_{1} \oplus \cdots \oplus G_{n}$ of orders $z_{i}, i=1, \cdots, n$, such that $z_{i} \mid z_{i+1}, 0<i<n$; this standard decomposition is not unique, even though the $z_{i}$ are. Since we want a description of $H(G, R)$ which is functorial in $G$ (and $R$ ), one is faced with two conflicting objectives: firstly, the final results must not depend on the given product decomposition; secondly, one practically has to use the structure theorem for $G$ to obtain any reasonably explicit description for $H(G, R)$. Thus one attempts to exploit a standard decomposition initially and then to eliminate the dependence of the direct sum decomposition by functorial methods. Thus our first step is to describe explicitly an

\footnotetext{
${ }^{1}$ Fellow of the Alfred P. Sloan Foundation.

2 NSF Senior Postdoctoral Fellow.
} 
augmented differential bigraded algebra $X$ over $\boldsymbol{Z}$ (which in fact is a bigraded differential Hopf algebra) such that $X$ depends on a standard direct sum decomposition of $G$ and that $X$ defines a resolution yielding the cohomology. Our choice is as follows.

Let $0 \rightarrow F \stackrel{\jmath}{\rightarrow} F \stackrel{\pi}{\rightarrow} G \rightarrow 0$ be a free resolution of $G$, where $F$ is free with generators $x_{i}, i=1, \cdots, n$ and $f x_{i}=z_{i} \cdot x_{i}, i=1, \cdots, n$. We let $\boldsymbol{Z}[G]$ denote the integral group ring (with $G$ written multiplicatively), and we form $X=Z[G] \otimes S F \otimes \wedge F$, where the tensor product is taken over $\boldsymbol{Z}, \wedge F$ is the exterior algebra over $\boldsymbol{Z}$ of the group $F$ all of whose elements have degree one, and where $S F$ is the symmetric algebra over $\boldsymbol{Z}$ of $F$ all of whose elements here have degree two; the gradation of $S F$ is considered to be the first gradation, and the one of $\Lambda F$ as the second. The augmentation is derived from the augmentation of $Z[G]$ in an obvious fashion. The differential on $X$ is defined as the sum of two differentials $d$ and $\partial$, which are characterized by the fact that they are derivations and $z[G]$-module morphisms, and satisfy $d\left(1 \otimes x_{i} \otimes 1\right)=\tilde{z}_{i} \otimes 1 \otimes x_{i}, \quad$ where $\tilde{z}_{i}=\pi\left(x_{i}\right)+\pi\left(x_{i}\right)^{2}+\ldots$ $+\pi\left(x_{i}\right)^{z i} \in Z[G], d\left(1 \otimes 1 \otimes x_{i}\right)=0, \quad \partial\left(1 \otimes x_{i} \otimes 1\right)=0$ and $\partial\left(1 \otimes 1 \otimes x_{i}\right)$ $=\left(\pi\left(x_{i}\right)-1\right) \otimes 1 \otimes 1$ for $i=1, \cdots, n$. What we have constructed is a particular Koszul complex [8, p. 204]; it may very well be equivalent to a complex indicated by Tate [10], and the triple $(Z[G] \otimes \wedge F, S F, X)$ is an acyclic construction in the sense of Cartan [2, p. 3-07]. It is however not a "special construction" $[2$, p. 4-05] but rather resembles certain particular constructions given by Cartan $[2$, p. 6-09 ff], although the choice of the symmetric algebra $S$ in place of a polynomial algebra with divided powers seems to set it off from Cartan's complexes. In any event, $0 \leftarrow Z \leftarrow X$ is a finitely generated $Z[G]$-free resolution of $\boldsymbol{Z}$ when $X$ is given the total degree. For cyclic $G$, we obtain the familiar resolution which is to be found in all textbooks. If we abbreviate the functor $\operatorname{Hom}_{Z[G]}(-, R)$ with $K$, where $G$ is still allowed to operate on $R$, then we have $H(G, R)=\operatorname{Ext}_{\boldsymbol{Z}[G]}(Z, R)$ $=H(K(X))$. Since there are natural $R$-module morphisms $K(X) \otimes_{R} K(X) \rightarrow K(X \otimes X) \rightarrow K(X)$ (using the comultiplication on $X)$, we may utilize the standard morphism $H(K(X)) \otimes_{R} H(K(X)$ ) $\rightarrow H(K(X) \otimes K(X))$ to derive a morphism $H(G, R) \otimes_{R} H(G, R)$ $\rightarrow H(G, R)$ which is exactly the cup product. Let us now assume that $G$ acts trivially on $R$. Then there is a natural isomorphism $K(X)$ $\rightarrow S_{R} \operatorname{Hom}(F, R) \otimes_{R} \Lambda_{R} \operatorname{Hom}(F, R)$, where the symmetric algebra and the exterior algebra are taken over $R$; the differential of $K(X)$ now gets transported into a differential $D$ of bidegree $(2,-1)$ which is characterized by the fact that it is a derivation and satisfies $D(\phi \otimes 1)$ $=0$ and $D(1 \otimes \phi)=(\phi \circ f) \otimes 1$ for all $\phi \in \operatorname{Hom}(F, R)$ (with $f$ from the 
free resolution of $G$ ). Thus we note that $K(X)$ is the $E_{2}$ term of a spectral algebra and it is of the following special type: If $\psi: A \rightarrow B$ is a morphism of $R$-modules, we let $E_{2}(\psi)$ denote the bigraded Hopf algebra $S_{R} B \otimes_{R} \Lambda_{R} A$ with a differential $D=D_{\psi}$ of bidegree $(2,-1)$ which is defined to be a derivation and to satisfy, $D(b \times 1)=0$ for $b \in B$ and $D(1 \otimes a)=\psi(a) \otimes 1$ for $a \in A$. Thus, if we define $E_{3}(\psi)$, to be the bigraded cohomology algebra of $E_{2}(\psi)$, we have finally arrived at the following formula for the graded cohomology ring $H(G, R)$.

$$
H(G, R) \cong E_{3}(\operatorname{Hom}(f, R)) \text {, with the total degree on } E_{3} \text {. }
$$

Admittedly, the spectral algebra $E_{r}(\operatorname{Hom}(f, R))$ is quite degenerate from a spectral algebra point of view, but we found it very useful to adopt the frame of mind suited to work with the initial term of a spectral algebra and its homology algebra, and in particular to have a special bigraded algebra which, relative to the total degree, is isomorphic to the wanted cohomology algebra.

However, in view of the primary objective, namely to find a functorial description of $H(G, R)$ as a ring, the isomorphism is not adequate, since the right side depends functorially on $f$ and not on $G$; it is true that $G$ depends on $f$ since $G=\operatorname{coker} f$, but the converse is incorrect. However, as a first step in the elimination of $f$, one can show the following

Proposition 1. The edge terms of $E_{3}(\operatorname{Hom}(f, R))$ depend functorially on coker $f=G$ alone and not on $f$. The horizontal edge term is naturally isomorphic to $S_{R} \operatorname{Ext}(G, R)$, the vertical edge term is naturally isomorphic to $\operatorname{Hom}(\Lambda G, R)$.

Thus, by Proposition 1 and (1) we obtain two natural coretractions of graded $R$-algebras.

$$
\begin{aligned}
& S_{R} \operatorname{Ext}(G, R) \rightarrow H(G, R), \\
& \operatorname{Hom}(\Lambda G, R) \rightarrow H(G, R) .
\end{aligned}
$$

There is some evidence that (3) is the right inverse of the morphism obtained via the universal coefficient theorem from a monomorphism $\Lambda G \rightarrow H_{*}(G, R)$ described by Eilenberg and MacLane [4, II, 19.3], although a direct verification may be intricate. The morphism (2) seems to be new. While $\operatorname{Hom}\left(\wedge^{n} G, R\right), n>0$, is frequently zero (e.g. when the additive group of $R$ is torsion free), this is never the case with $\operatorname{Ext}(G, R)$. So $H(G, R)$ is a $S_{R} \operatorname{Ext}(G, R)$-module in a natural way. The morphisms (2) and (3) together yield a natural morphism of bigraded algebras 


$$
\omega_{G, R}: S_{R} \operatorname{Ext}(G, R) \otimes_{R} \operatorname{Hom}(\Lambda G, R) \rightarrow H(G, R) .
$$

In general, $\omega_{G, R}$ is neither injective nor surjective (take $G$ $=\boldsymbol{Z}(2) \oplus \boldsymbol{Z}(4), \boldsymbol{R}=\boldsymbol{Z} / 4 \boldsymbol{Z}$ and consider $\left.\omega_{G, R}^{21}\right)$. However, we have the following results about (4), the first of which is related to a result of Cartan's obtained by different methods [2, p. 9-08].

Proposition 2. If $R$ is a field, then $\omega_{G, R}$ is an isomorphism of Hopf algebras. Also, if $K$ is the prime field of $R$ then $H(G, R)$ is isomorphic to $R \otimes S \operatorname{Tor}(\hat{G}, K) \otimes \Lambda \operatorname{Tor}(\hat{G}, K)$ where the tensor products, $S$ and $\Lambda$, are taken either over $\boldsymbol{Z}$ or over $K$, and where $\hat{G}$ is the character group of $G$.

Proposition 3. If $R=\boldsymbol{Z} / m \boldsymbol{Z}$ and $G \cong \boldsymbol{Z}\left(m^{\prime}\right)^{n}$ with $m \mid m^{\prime}$, then $\omega_{G, R}$ is an isomorphism.

Proposition 4. If $R=\boldsymbol{Z} / m \boldsymbol{Z}$ and $m \mid z_{1}$ (where $z_{1}$ is the order of the smallest cyclic group in the standard direct sum decomposition of $G$ ), then $\omega_{G, R}$ is an isomorphism.

Clearly Proposition 3 is a special case of Proposition 4, but the former is particularly instructive since here one can obtain $H(G, \boldsymbol{Z})$ as a subring of $H(G, R)$ (except, for degree 0 ) in the following fashion: Since $\operatorname{Hom}(\Lambda G, R) \cong \Lambda \operatorname{Hom}(G, R)$ in this case, by Proposition 4 we may identify $H(G, R)$ with $E_{2}(i)$ where $i$ is the identity morphism of $\operatorname{Hom}(G, R)$; under this identification, the Bockstein derivation on $H(G, \boldsymbol{Z} / m \boldsymbol{Z})$ becomes identified with the differential $D_{\boldsymbol{i}}$ of the spectral algebra $E_{2}(i)$ as described above. Finally, $\boldsymbol{Z} \oplus \operatorname{im} D_{\boldsymbol{i}}$ (with $\boldsymbol{Z}$ in degree 0 ) as a graded algebra is isomorphic to $H(G, \boldsymbol{Z})$. This observation is typical for the utilization of the Bockstein morphism in the present context.

After all this, an explicit description of the ring $H(G, Z)$ is still missing, but we do obtain partial results. In this case the vertical edge term $\operatorname{Hom}(\Lambda G, Z)$ of $E_{3}(\operatorname{Hom}(f, R))$ is zero (except in bidegree $(0,0)$ ). However, the third vertical column in $E_{3}(\operatorname{Hom}(f, R))$ (which is the first nontrivial one) takes over and brings significant information. In fact, let $M^{i}=E^{2, i-2}(\operatorname{Hom}(f, R))$ and $M=M^{2}+M^{3} \cdots$.

Proposition 5. The submodule $M$ of $E_{3}(\operatorname{Hom}(f, R))$ depends only on coker $f=G$ and not on $f$. The group $M^{i+1}$ is naturally isomorphic to $\Lambda^{i} \hat{G}$, where $\hat{G}$ is the character group of $G$. Specifically, if $H^{i}(G, Q / Z)$ $\rightarrow H^{i+1}(G, Z)$ is the connecting morphism deduced in the long cohomology sequence, and $\operatorname{Hom}(\Lambda G, Q / Z) \rightarrow H(G, Q / Z)$ is the natural morphism of graded groups (3) (which prevails even if $R$ is just a group and not a ring), then the composition $\operatorname{Hom}\left(\Lambda^{i} G, Q / Z\right) \rightarrow H^{i+1}(G, Z)$ is injective 
and has $M^{i+1}$ as its image, if $H(G, Z)$ and $E_{3}(\operatorname{Hom}(f, Z))$ are identified under (1).

Proposition 6. Under the conditions of Proposition 5, the ring $H(G, Z)$ is generated by the submodule $Z+M$ and by no proper submodule of $\boldsymbol{Z}+M$. Also $H(G, Z)$ is a torsion-free $S \hat{G}$-module via (2) (where $\operatorname{Ext}(G, Z)$ is naturally isomorphic to the character group $\hat{G}$ ) and as an $S \hat{G}$-module is generated by $Z+M$.

Note that if in the standard decomposition of $G$ we have $n$ summands, then elements of degree as high as $n+1$ are needed to generate $H(G, Z)$ as a ring. (This would seem to contradict an unproved assertion in [5], according to which $H(G, Z)$ is generated by the elements of degree 3 or less, regardless of $G$.)

The authors are happy to acknowledge valuable correspondence with S. MacLane who, in particular, pointed out to them some of the pertinent literature.

\section{REFERENCES}

1. H. Cartan, La transgression dans un groupe de Lie et dans un espace fibrê principal, Colloque de Topologie, Bruxelles, 1950, pp. 57-71.

2. - Algèbres d'Eilenberg-MacLane et homotopie, Séminaire Henri Cartan, 7 e annèe, 1954/55, Paris.

3. H. Cartan and S. Eilenberg, Homological Algebra, Princeton Univ. Press, Princeton, N. J., 1956.

4. S. Eilenberg and S. MacLane, On the groups $H(\pi, n)$. I, II, III, Ann. of Math. (2) 58 (1953), 55-106; (2) 60 (1954), 49-139; (2) 60 (1954), 513-557.

5. L. Evens, The cohomology ring of a finite group, Trans. Amer. Math. Soc. 101 (1961), 224-239.

6. K. H. Hofmann and P. S. Mostert, The cohomology of compact abelian groups, Bull. Amer. Math. Soc. 74 (1968), 975-978.

7. J. L. Koszul, Sur un type d'algèbres differentielles en rapport avec la transgression, Colloque de Topologie, Bruxelles, 1950, pp. 73-81.

8. S. MacLane, Homology, Springer-Verlag, Berlin, 1963.

9. J. Schafer, On the homology ring of an abelian group, Dissertation, University of Chicago, Chicago, Ill., 1965.

10. J. Tate, Homology of Noetherian rings and local rings, Illinois J. Math. 1 (1957), 14-27.

Tulane University, New Orleans, Louistana 70118 and

Institute for Advanced Study, Princeton, New Jersey 08540 